\title{
EDITORIAL
}

\section{Optimising treatment pathways for borderline and locally advanced pancreatic cancer: an adaptive personalised} approach

The treatment paradigm for borderline and locally advanced pancreatic cancer is evolving with an increased shift towards utilising systemic chemotherapy and chemoradiation to potentially facilitate more curative resections. This has been driven by the improved outcomes from the use systemic combination chemotherapy on its own, or sequentially with chemoradiation, resulting in improved resection rates and survival outcomes.

British Journal of Cancer (2021) 125:1179-1180; https://doi.org/10.1038/s41416-021-01416-8

\section{MAIN}

The best outcomes for patients with non-metastatic pancreatic cancer remain in those who have successful surgical resections; but only $1: 10^{1}$ patients may have this option at the time of diagnosis. Recently, the benefit of neoadjuvant treatments in improving clinical outcomes has been demonstrated ${ }^{2,3}$ for borderline resectable pancreatic cancer. However, the evidence for the use of sequential therapy for locally advanced disease is under review, with sequential combination chemotherapy followed by concurrent chemoradiation ${ }^{4}$ or newer radiation techniques e.g. Stereotactic Body Radiotherapy (SBRT). ${ }^{5}$ These approaches are being explored in clinical trials with a view to improving clinical outcomes for these patients. This may result in increasing the number of patients who become suitable for curative resections, and subsequently further improve their survival outcomes.

In the paper by the AGEO group, which accompanies this editorial, we see the tantalising prospect of improved clinical outcomes with expert MDT driven use of sequential systemic and radiation therapy. The authors use follow on chemoradiation and observed more curative surgery for patients with borderline resectable and locally advanced pancreatic cancer. In this study of more than 300 patients, the authors consistently applied the NCCN definition for borderline and locally advanced pancreatic cancer. Crucially, the authors systematically and consistently utilised expert specialist pancreatic MDT to formulate individual patient specific treatment plans and produced "real-world" data to support the use of optimal sequential therapy. They observed an improvement in survival outcomes with patients receiving chemotherapy having a median overall survival of 16.8 months and those having chemoradiation achieving a median overall survival of 21.8 months. Patients who then proceeded to have a surgical resection following chemotherapy alone or sequentially with chemoradiation derived the best survival outcomes with a median survival which has not been reached. This was achieved without excess $\mathrm{G} 3 / 4$ toxicity rates. These findings require further exploration within clinical trials to determine the optimal sequential therapeutic options for these patients.

Nevertheless, the retrospective nature of the study and clinician dependent selection of the treatment pathway does confer bias which needs to be taken into consideration in the interpretation of this study. The use of radiation therapy in this study which led to improved outcomes is in contrast with the findings from the recent Alliance AO20501 study ${ }^{6}$ which had poorer outcomes in the cohort of patient treated with sequential Folfirinox and SBRT. It remains to be ascertained as to why this effect may have been encountered. Possible explanations may be due to the treatment volume effect utilised with SBRT and / or the effect of fractionation schedule used. In both this AEGO study and the Dutch PREOPANC trial, $^{7}$ the use of conventional or moderately hypofractionated radiation therapy concurrent with chemotherapy appeared to lead to improved outcomes. Although the role of sequential Folfirinox and SBRT in the context of borderline resectable is now much less certain, ${ }^{6}$ the role of SBRT following multiagent systemic chemotherapy in locally advanced pancreatic cancer may have some benefit for patients where surgery is not an option as highlighted by a recent cohort study ${ }^{5}$ which demonstrated improved local control ( 2 year local failure rate of $32.8 \%$ ) and survival outcomes (median survival of 26.8 months). Further studies exploring the utility of non SBRT based radiation therapy, sequentially with optimal combination chemotherapy are required in the preoperative setting; and the potential role of SBRT and non SBRT chemoradiation schedules following systemic chemotherapy in the locally advanced setting are also required.,

This AEGO study does highlight the need for more precision, in both patient selection and adapting the treatment pathways accordingly, led by expert pancreatic MDTs, to improve outcomes and facilitate surgical resection whenever possible. The Primus 002 trial $^{8}$ which a part of the UK Precision Panc Platform trial, is such a biomarker driven trial which aims to potentially identify treatments based on patients DDR (deficient in DNA repair)status. Various groups are also looking to personalise and adapt treatments by standardising treatment algorithms and applying consistent patient stratification, for example the proposed NCRI pancreatic cancer Umbrella study, ${ }^{9}$ should enable improved selection of treatment algorithms for specific patient cohorts, leading to optimised use of systemic and radiation therapy combinations when indicated and ultimately, lead to further improvement in patient outcomes for patients with nonmetastatic pancreatic cancer. 
1180

\section{ACKNOWLEDGEMENTS}

Not applicable

\section{AUTHOR CONTRIBUTIONS}

G.R. designed and wrote the first draft of the manuscript. P.G. reviewed the relevant data discussed and contributed to manuscript writing. Both authors edited the manuscript and approved the manuscript for final publication.

\section{ADDITIONAL INFORMATION}

Ethics approval and consent to participate No formal ethics approval was required for this manuscript, as per the local ethics committee and national guidance. No consent to participate was required.

Consent to publish Not applicable.

Data availability Not applicable.

Competing interests The authors declare no competing interests.

Funding information The authors received no specific funding for this work.

Publisher's note Springer Nature remains neutral with regard to jurisdictional claims in published maps and institutional affiliations.

Ganesh Radhakrishna ${ }^{1}$ and Paula Ghaneh ${ }^{2}$ ${ }^{1}$ Consultant Clinical Oncologist, The Christie Hospital, Manchester, UK and ${ }^{2}$ Professor of Surgery, Department of Molecular and Clinical Cancer Medicine, University of Liverpool, Liverpool, UK Correspondence: Ganesh Radhakrishna (g.radhakrishna@nhs.net)

\section{REFERENCES}

1. Pancreatic Cancer UK website. https://www.pancreaticcancer.org.uk/what-we-do/ media-centre/pancreatic-cancer-statistics/ (2021).

2. Ghaneh, P., Palmer, D. H., Cicconi, S., Halloran, C., Eirini Psarelli, E., Rawcliffe, C. L. et al. and European Study Group for Pancreatic Cancer (ESPAC). ESPAC-5F: Fourarm, prospective, multicenter, international randomized Phase II trial of immediate surgery compared with neoadjuvant gemcitabine plus capecitabine (GEMCAP) or FOLFIRINOX or chemoradiotherapy (CRT) in patients with borderline resectable pancreatic cancer. J. Clin. Oncol. 38, 4505-4505 (2020).

3. Grose, D., McIntosh, D., Jamieson, N., Carter, R., Dickson, E., Chang, D. et al. The role of induction chemotherapy + chemoradiotherapy in localised pancreatic cancer: initial experience in Scotland. J. Gastrointest. Oncol. 8, 683-695 (2017).

4. Hurt, C. N., Falk, S., Crosby, T., McDonald, A., Ray, R., Joseph, G. et al. Long-term results and recurrence patterns from SCALOP: a Phase II randomised trial of gemcitabine- or capecitabine-based chemoradiation for locally advanced pancreatic cancer. Br J Cancer 116, 1264-1270 (2017).

5. Reyngold M., O'Reilly E. M., Varghese A. M., Fiasconaro M., Zinovoy M., Rommesser P. B. et al. Association of ablative radiation therapy with survival among patients with inoperable pancreatic cancer. JAMA Oncol. https://doi.org/10.1001/ jamaoncol.2021.0057 (2021).

6. Katz, M. H., Shi, Q., Meyers, J. P., Herman, J. M., Choung, M. et al. Alliance A021501: preoperative $\mathrm{mFOLFIRINOX}$ or $\mathrm{mFOLFIRINOX}$ plus hypofractionated radiation therapy (RT) for borderline resectable (BR) adenocarcinoma of the pancreas. J. Clin. Oncol. 39, 377-377 (2021).

7. Versteijne, E., Suker, M., Groothuis, K., Akkermans-Vogelaar, J. M., Besselink, M. G., Bonsing, B. A. et al. Preoperative chemoradiotherapy versus immediate surgery for resectable and borderline resectablepancreatic cancer: results of the Dutch Randomized Phase III PREOPANC Trial. J.Clin. Oncol. 38, 1763-1773 (2020).

8. Grose, D. B., McKay, C. J., Cooke, S., Graham, J. S., Duthie, F., Jamieson, N. et al. PRIMUS-002: a multicentre, open-label, Phase II study examining FOLFOX and nabpaclitaxel (FA) and nab-paclitaxel and gemcitabine (AG) as neoadjuvant therapy for (borderline) resectable pancreatic cancer (PC), focusing on biomarker and liquid biopsy development. J. Clin. Oncol. 37, 4166 (2019).

9. NCRI Upper gastrointestinal Group report 2019-20. https://www.ncri.org.uk/wpcontent/uploads/NCRI-Upper-GI-Group-Annual-Report-2019-20.pdf (2021). 